- Original Article

\title{
Comparison of Physiotherapy Approaches in Low Back Pain: A Randomized Controlled Trial
}

\author{
Gamze Senbursa', Nihan Ozunlu Pekyavas², Gul Baltaci ${ }^{3, *}$ \\ ${ }^{1}$ Anima Rapha Center of Manual Therapy and Reflexology, Ankara, Turkey \\ ${ }^{2}$ Department of Physiotherapy and Rehabilitation, Faculty of Health Sciences, Baskent University, Ankara, Turkey \\ ${ }^{3}$ Department of Physiotherapy and Rehabilitation, Private Guven Hospital, Ankara, Turkey
}

Background: The purpose of this study was to compare the effectiveness of rehabilitation approaches in individuals with low back pain (LBP) on pain, spinal mobility, disability, and muscular strength.

Methods: Ninety volunteers were included and divided into four groups depending on the rehabilitation approach: group 1, soft tissue mobilization techniques and stabilization exercises ( $\mathrm{n}=24 ; 11$ females [F], 13 males [M]); group 2 , Kinesio Taping and stabilization exercises $(\mathrm{n}=24 ; 12 \mathrm{~F}, 12 \mathrm{M})$; group 3 , stabilization exercises $(\mathrm{n}=22 ; 11 \mathrm{~F}, 11 \mathrm{M})$; and group 4, reflex therapy and stabilization exercises $(\mathrm{n}=20 ; 10 \mathrm{~F}, 10 \mathrm{M})$. Visual Analog Scale for pain intensity, an isokinetic evaluation for strength at $60 \%$ s and a side-plank position test for trunk stabilization were measured before and assessed at the beginning, after a 4-week treatment and during 4 weeks of follow-up. The functional status was evaluated with the Oswestry Disability Index.

Results: Individuals in all groups showed similar decrease in pain after the treatment and at 1-month follow-up, but there were no significant differences in pain levels between the groups $(\mathrm{P}<0.05)$.

Conclusion: All therapeutic approaches were found to be effective in diminishing pain and thus helpful in increasing strength and stabilization in patients with LBP.

Keywords: Low Back Pain; Musculoskeletal Manipulations; Reflexotherapy; Exercise; Muscle Strength; Spinal Manipulation 


\section{INTRODUCTION}

Today, there is a great emphasis on evidence-based approaches in low back pain (LBP) for public health to reduce medical costs covering back injuries. LBP is an important issue due to its high occurrence, increased treatment costs, and the effect on the quality of life in a large population throughout the lifespan. ${ }^{1)}$

LBP is defined as pain in the lower portion of the back, which is persistent for at least 12 weeks without any specific cause. It is one of the most common musculoskeletal disorders and it has been stated that over $80 \%$ population worldwide will experience a LBP episode. ${ }^{2)}$ This pathology is related to functional limitation, disability, and pain in developed countries, which is associated with serious economic impact because of received health care and work absenteeism. The health care and social costs associated with LBP have been estimated to be between $\$ 100$ to $\$ 200$ billion annually with a majority of these costs due to lost wages and decreased productivity. ${ }^{3)}$

Management of LBP aims to control pain, return patients to work at early stages and prevent chronic disability. Pain relief is provided by conservative treatment, drugs administration including nonsteroid anti-inflammatory drugs, thermotherapy, and electrotherapy, and functional restoration of the spine is achieved through exercise. ${ }^{4-6)}$ Manual therapy, ${ }^{7-9)}$ reflex therapy, ${ }^{10,11)}$ and Kinesio Taping ${ }^{12-14)}$ techniques are some of the passive treatment methods for reducing pain and improving biomechanics of the lumbar region.

As a part of the active treatment, core stabilization exercises are one of the exercise programs for chronic LBP management. ${ }^{15)}$ As core muscles work in an effective and coordinated fashion, excessive movement of the pelvis and spine during movement of the limbs is prevented, and abnormal and excessive loads can be controlled and a proper posture maintained. ${ }^{16,17)}$

One of the newly developed treatment methods is reflex therapy, which is defined as the power of the body to heal itself with the help of manual deep stroking techniques applied to specific areas of the body, especially the feet. ${ }^{3)}$ Several researchers have studied this subject and revealed conflicting results. According to some research, reflex therapy appears to offer promise as a treatment in the management of $\mathrm{LBP}^{10,18,19)}$

Kinesio Tape application is similar to corrective and basic techniques such as application/placement of hands onto the patient. The Kinesio Taping method allows the fascia to release tension and muscles to move properly. ${ }^{12)}$ Kinesio Taping methods applied to LBP have been studied by Paoloni et al. ${ }^{20}$ and the researchers stated that Kinesio Taping led to pain relief and lumbar muscle function normalization shortly after its application. Moreover, Castro-Sanchez et al. ${ }^{13)}$ reported that Kinesio Taping reduced disability and pain in people with chronic non-specific LBP, but these effects seemed too weak to be clinically worthwhile. On the other hand, Kim et al. ${ }^{14)}$ showed that Kinesio Taping was more efficacious than placebo in patients with a non-specific LBP.

Although there are numerous studies on LBP management, there are no studies comparing the effects of most popular treatment ap- proaches for rehabilitation. Therefore, the aim of our study was to compare the effectiveness of four physical therapy approaches including soft tissue manual therapy, Kinesio Taping techniques, stabilization exercises, and reflex therapy for treating lumbar disc pathologies without neurological deficits.

\section{METHODS}

\section{Study Design}

The study was a randomized controlled trial with a blind evaluator and a follow-up of 4 weeks. The study was approved by the Human Ethics Committee of the University (NCT04061759) and conducted in accordance with the Declaration of Helsinki, good clinical practices, and applicable laws and regulations and meets the CONSORT guidelines standards. ${ }^{21)}$ Informed consent was obtained from all the participants and the rights of the participants were protected.

Ninety patients (44 females, 46 males) with LBP were randomly assigned to one of the four groups using an online random allocation software program (GraphPad Software QuickCalcs; GraphPad Software Inc., La Jolla, CA, USA). The groups were as follows: group 1, soft tissue mobilization techniques and stabilization exercises $(n=24)$; group 2, Kinesio Tape and stabilization exercises ( $\mathrm{n}=24)$; group 3, stabilization exercises ( $n=22)$; and group 4 , reflex therapy and stabilization exercises $(n=20)$. Groups 1,2 , and 4 started treatment twice a week for 4 weeks after the first evaluation. Group 3 followed home exercise program after the first evaluation. All groups were assessed anew after a 4-week treatment (end of treatment) and 4 weeks after the completion of the treatment (follow-up). One therapist (G.S.) who has international qualifications and certifications in Kinesio Taping from Kinesio Taping Association International, reflexotherapy from Barcelona Reflexotherapy Institute, manual therapy from Cyriax Orthopaedic Manual Therapy, and stabilization exercises from TheraBand Academy received all patients. The others were responsible for data analysis before and after the testing.

All patients who were admitted to the Department of Physiotherapy and Rehabilitation of the University with LBP were included in the study. The sociodemographic characteristics (age, sex, occupation, marital status, disease duration, previous treatments) of the patients as well as the severity of their pain were recorded. To be enrolled in the present study, participants had to meet the following inclusion criteria: (1) a history of LBP exceeding 12 weeks (with lumbar disc pathology including bulging or protrusion diagnosed by clinical tests and magnetic resonance imaging); (2) age between 18 and 50 years; (3) the willingness to comply with any one of the randomly chosen treatment programs; (4) have a minimum pain level (at activity) of 3 out of 10 on Visual Analog Scale (VAS) and had LBP for at least 3 months; and (5) the written informed consent of the patient.

The exclusion criteria were: (1) presence of radiculopathy or other damages to the spine such as fractures, stenosis, or tumors; (2) not habitual exercise practitioners; (3) cardiovascular or systemic diseases or any condition which contraindicated or made the exercise training 
impossible; (4) neurological deficit; (5) presence of a psychiatric disorder which might affect the compliance and the assessment of symptoms; (6) history of spinal surgery; (7) pregnancy; (8) inflammatory, infectious, or malignant diseases of the vertebra; (9) severe structural deformity; and (10) physiotherapy previously received for at least 12 months or any surgery affecting the lumbar region. The details of included and excluded subjects through final data analysis are provided in Figure 1 as flowchart.

\section{Assessments and Outcome Measurements}

\section{1) Pain}

All patients were assessed with the VAS for pain intensity at rest, at night and during activity. VAS is a 100-mm line with no marks along it, anchored with the words "no pain" on one side and "the most severe pain" on the other. The subjects were instructed to place a mark along the line at a level representing the intensity of their pain. ${ }^{22)}$

\section{2) Isokinetic strength}

All patients were assessed with isokinetic testing for strength at $60 \%$ s. Isokinetic testing is commonly used for testing and training of patients in clinics. It calculates the muscle power at the full range of motion, providing the opportunity to act at the angular velocity ${ }^{23)}$ An IsoMed 2000 (D\&R GmbH, Hemau, Germany) was used bilaterally for isokinetic evaluation of hip flexion and abduction at $60^{\circ} \%$. After proper positioning, the patient was asked to push the force arm of the system as strongly as possible at $60^{\circ} / \mathrm{s}$ angular velocity in the flexion and ab- duction directions. The peak torque and total work values were recorded.

\section{3) Stabilization}

A side-plank test was used for evaluation of trunk stabilization. This test is one of the most functional stabilization tests and examines trunk strength, endurance, and stabilization during synchronized extremity movements. The lateral core muscles are assessed but oblique abdominal muscles and hip flexors are examined. Patients are positioned in side lying, with $90^{\circ}$ elbow flexion, $60^{\circ}$ shoulder abduction, legs extended, and whole body aligned. After trial repetition, patients are asked to raise their pelvis off the ground and stay in that position as long as possible without disrupting the smoothness of the motion. The test is finished when the position is disrupted or the patient falls. The time (in seconds) is recorded. ${ }^{24)}$

\section{4) Functionality}

The functional status was evaluated with the Oswestry Disability Index. Knowing the symptoms and disabilities of LBP patients provides valuable information for planning the treatment process. The Oswestry Disability Index has 10 main parameters; namely, pain intensity, self-care, lifting, walking, sitting, standing, sleep quality, sexual function, social life, and traveling. Each parameter is scored from 0 to 5 points. The degree of disability increases as the score increases. ${ }^{25}$ )

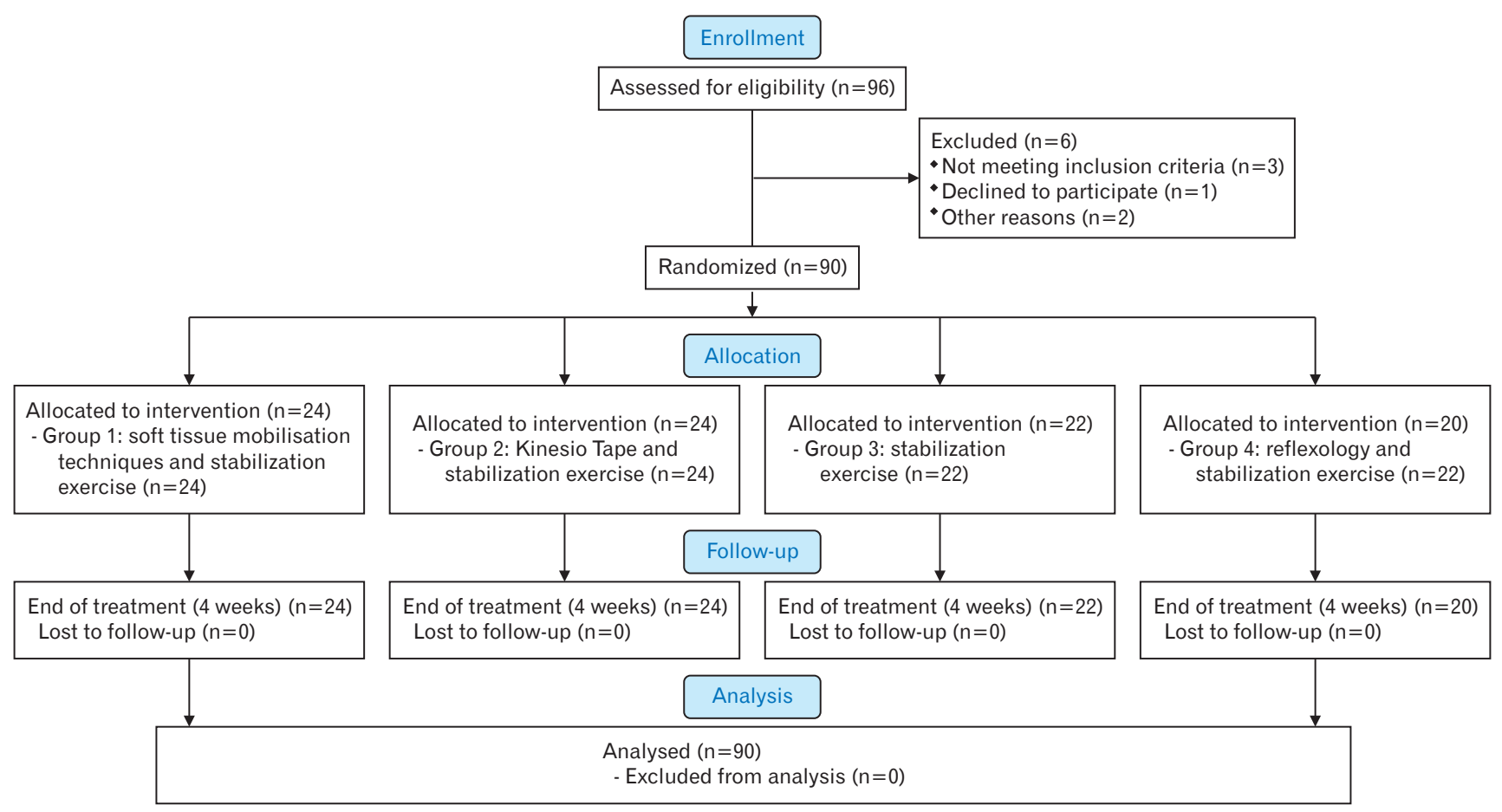

Figure 1. Flowchart of the study. 


\section{Treatment Approaches}

\section{1) Manual therapy}

The following manual therapy techniques were applied: (1) soft tissue mobilization; (2) pretzel maneuvers (Figure 2); (3) pelvis backwarddistraction; (4) trunk rotation; (5) multifidus mobilization; and (6) piriformis transverse friction massage. ${ }^{26)}$

\section{2) Reflex therapy}

Mobilization of each vertebra and pulls were applied from the medial side of the toe to the medial malleolus and to the heel by hand or with

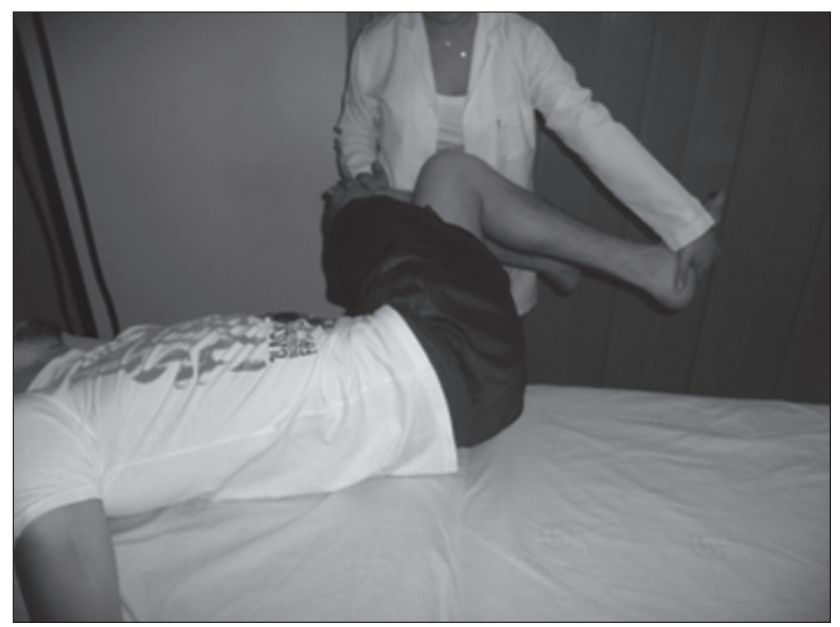

Figure 2. Pretzel maneuver. the help of an apparatus, including the cervical, thoracic, and lumbar spine reflex zones. Finally, the procedure was completed by making a V-shaped maneuver with a thumb in the direction of spinal nerve exits (Figure 3). ${ }^{19,27)}$

\section{3) Kinesio Taping technique}

The Kinesio Taping muscle technique, with $10 \%-25 \%$ of the stretch of the tape, was applied to the sacrospinalis, quadratus lumborum, gluteus, medius/maximus, and piriformis muscles (Figure 4), based on the weakness that patient's muscles had. Factors interfering with tape adhesion, such as sweat or hair, were removed before tape application.

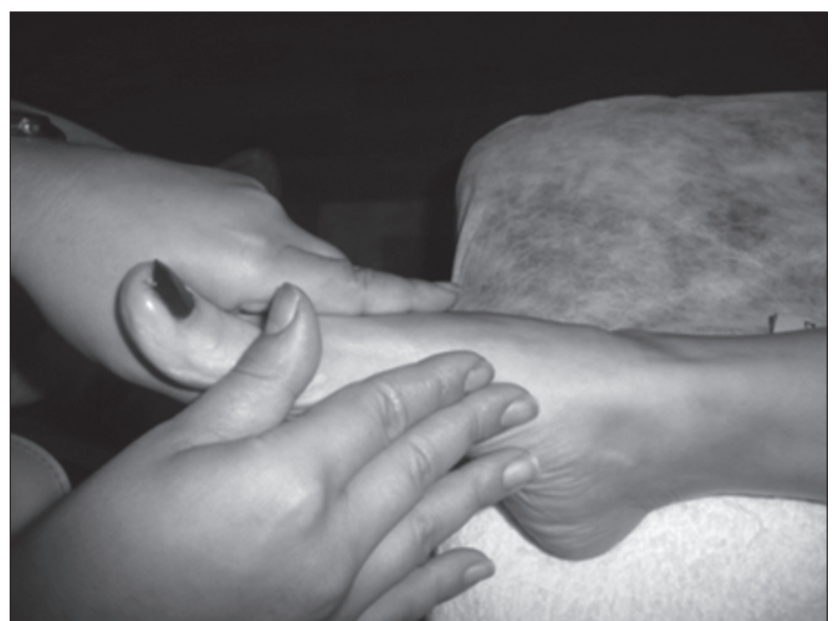

Figure 3. Reflex therapy application.
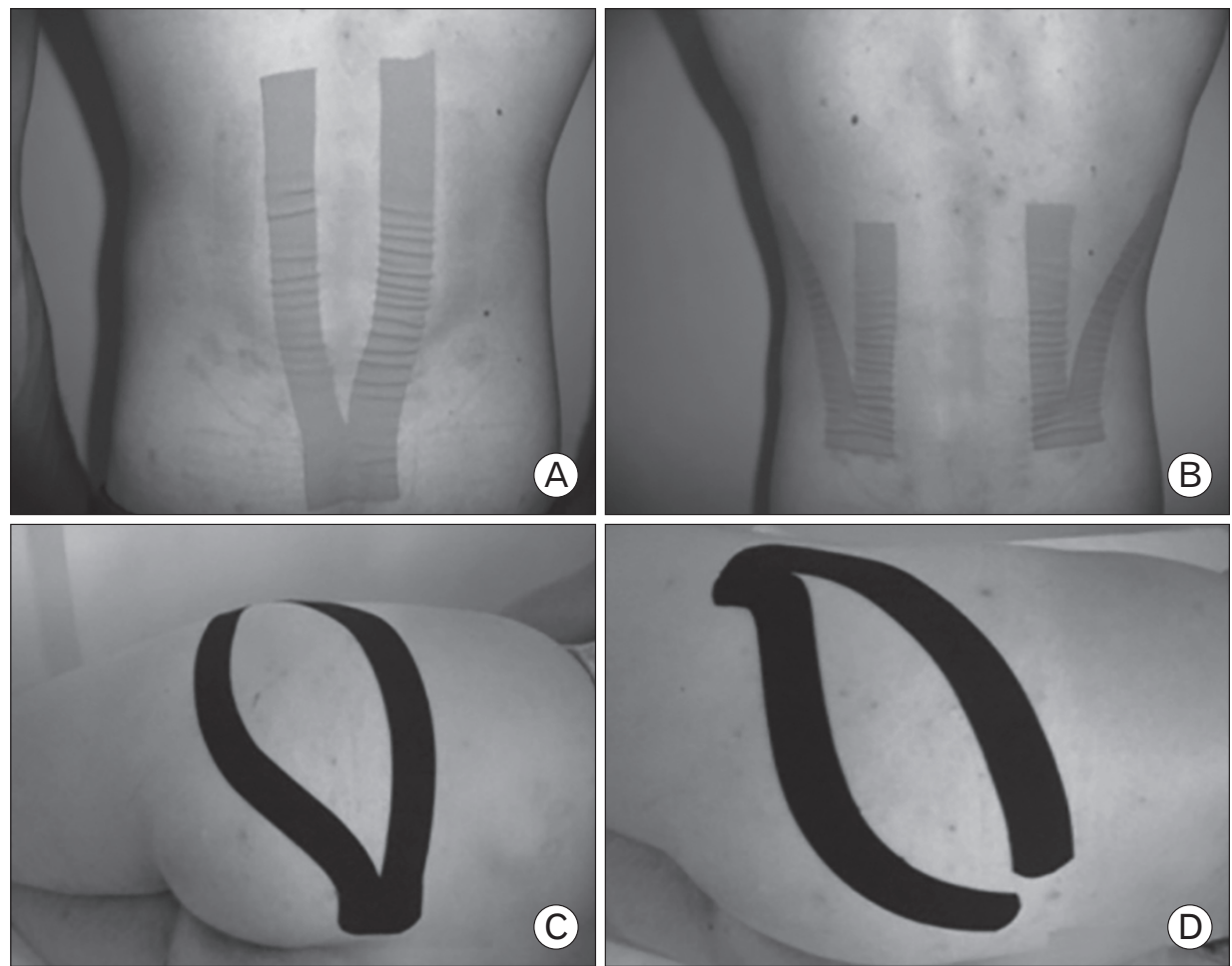

Figure 4. (A) Sacrospinalis, (B) quadratus lumborum, (C) gluteus medius/maximus, and (D) piriformis muscles Kinesio Taping muscle technique. 
The tape could stay in place for 3-5 days due to its water resistant and breathable properties. ${ }^{28)}$

\section{4) Stabilization exercises}

The core stabilization exercise treatment program consisted of the following exercises: ${ }^{29)}$ a posterior pelvic tilt exercise; lower abdominal muscle isometric strengthening; hip adductor muscle isometric strengthening; lumbar stabilization exercises with a Swiss ball; upper and lower abdominal muscle strengthening exercises with a Swiss ball; oblique abdominal muscle strengthening exercises with a Swiss ball; quadratus lumborum muscle stretching with a Swiss ball; back extensor muscle strengthening exercises with a Swiss ball; a slump exercise, lumbar lordosis exercises with a Swiss ball; bridge exercises with a Swiss ball; single leg bridge exercises on a Swiss ball; posture exercises; push-up exercises with a Swiss ball; and squat exercises with a Swiss ball (Figure 5).

\section{Statistical Analysis}

Data were analyzed using the IBM SPSS ver. 23.0 statistical package (IBM Corp., Armonk, NY, USA). The normal distribution of continuous variables was verified using the Kolmogorov-Smirnov test $(\mathrm{P}<0.05)$. Mean and standard deviations were calculated for each variable. Between-groups variables were compared using the Student t-test. A repeated measure analysis of variance with Bonferroni correction for
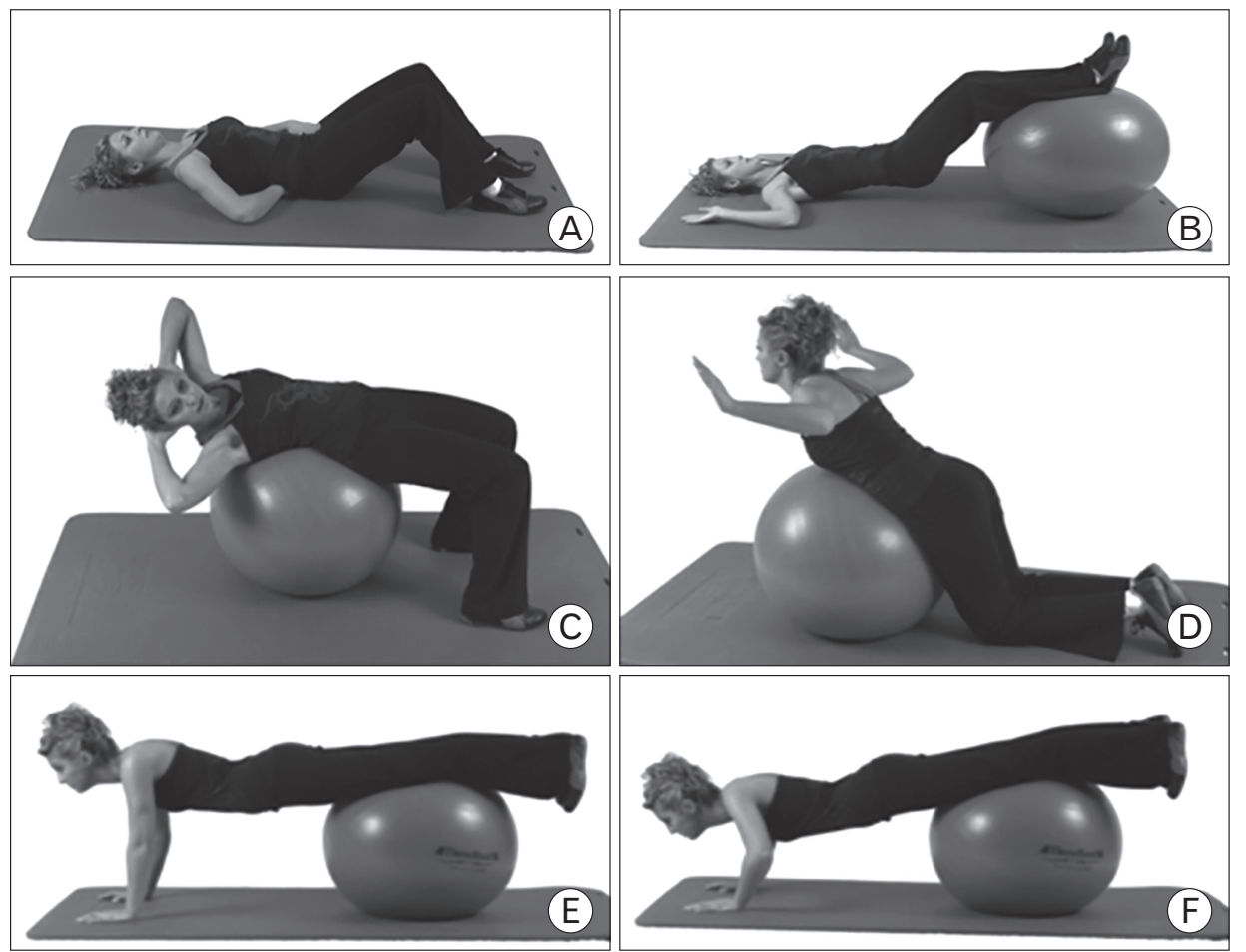

Figure 5. (A-F) Examples of stabilization exercises.

Table 1. Sociodemographic characteristics of participants

\begin{tabular}{|c|c|c|c|c|c|}
\hline Characteristic & Group $1(n=24)$ & Group $2(n=24)$ & Group $3(n=22)$ & Group $4(n=20)$ & P-value* \\
\hline Age (y) & $41.25 \pm 11.07$ & $45.33 \pm 8.49$ & $42.05 \pm 10.33$ & $42.55 \pm 12.45$ & 0.180 \\
\hline \multicolumn{6}{|l|}{ Sex } \\
\hline Female & $11(45.8)$ & $12(50.0)$ & $11(50.0)$ & $10(50.0)$ & \\
\hline Male & $13(54.2)$ & $12(50.0)$ & $11(50.0)$ & $10(50.0)$ & \\
\hline Height (cm) & $171.75 \pm 10.94$ & $167.29 \pm 9.41$ & $168.27 \pm 13.01$ & $170.50 \pm 11.00$ & 0.070 \\
\hline Weight (kg) & $75.29 \pm 13.27$ & $81.10 \pm 14.55$ & $79.30 \pm 11.18$ & $74.38 \pm 13.42$ & 0.095 \\
\hline Body mass index $\left(\mathrm{kg} / \mathrm{m}^{2}\right)$ & $25.46 \pm 2.75$ & $29.02 \pm 4.04$ & $28.24 \pm 3.83$ & $25.64 \pm 4.10$ & 0.091 \\
\hline Pain duration (mo) & $33.1 \pm 13.27$ & $38 \pm 31.89$ & $40.2 \pm 12.47$ & $41.5 \pm 31.20$ & 0.077 \\
\hline \multicolumn{6}{|l|}{ Tobacco use } \\
\hline Yes & $6(25.0)$ & $5(20.8)$ & $4(18.2)$ & $5(25.0)$ & 0.090 \\
\hline No & $18(75.0)$ & 19 (79.2) & 18 (81.8) & $15(75.0)$ & 0.965 \\
\hline
\end{tabular}

Values are presented as mean \pm standard deviation or number (\%). Group 1: soft tissue mobilization+stabilization exercise; group 2: Kinesio Tape+stabilization exercise; group 3: stabilization exercise; and group 4: reflexology+stabilization exercise.

${ }^{*} \mathrm{P}<0.05$. 


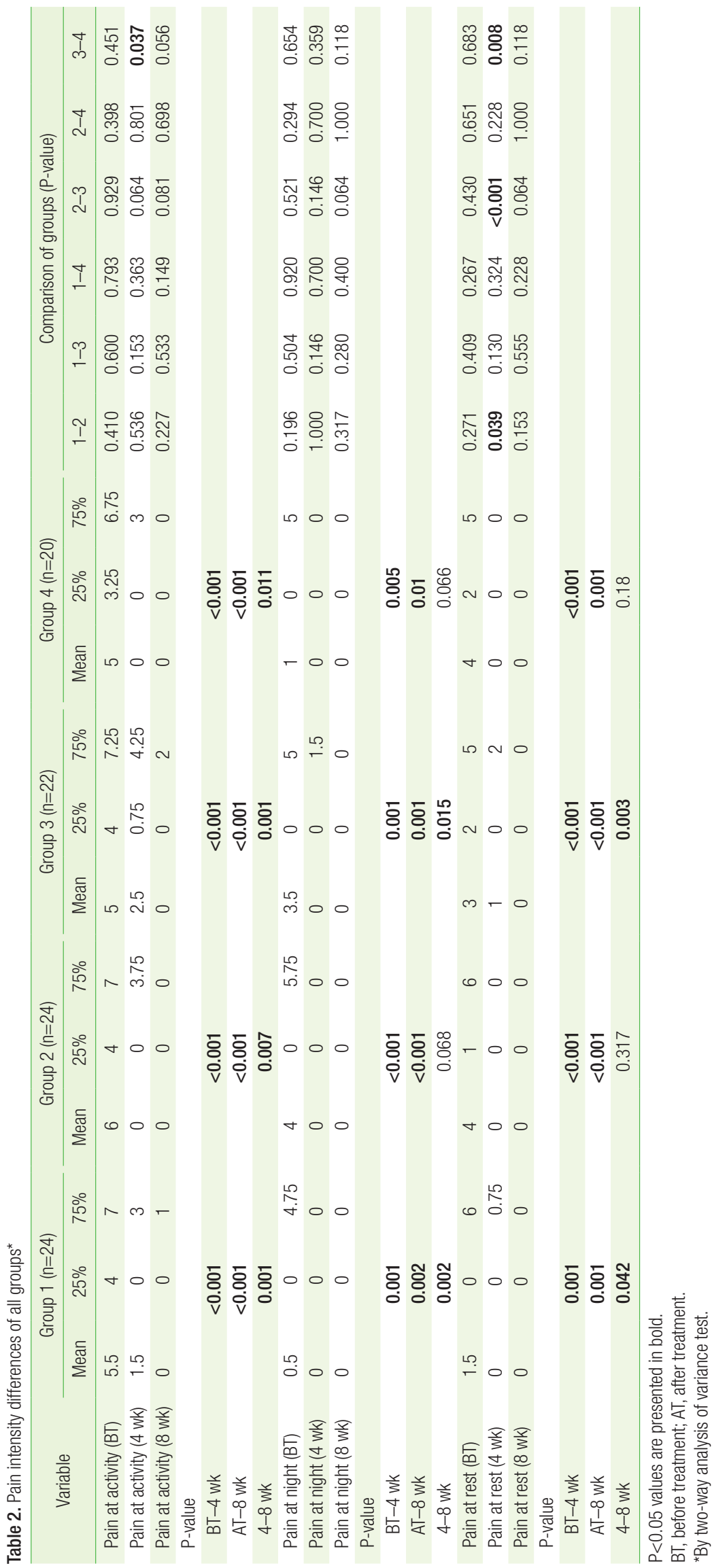


post-hoc analyses was used. The Bonferroni value was adjusted to 0.025 . The effectiveness of treatments in the groups before the treatment, after the treatment and at 1-month of the follow-up was compared by means of the multiple comparison tests using the Friedman's bi-directional variance analysis. ${ }^{30)}$ Sample size calculation was performed using ENE3.0 (GlaxoSmithKline, Brentford, UK) based on related studies that examined the effects of reflex therapy on LBP assuming a $20 \%$ improvement in the pain VAS score. The sample size calculation was performed with $80 \%$ power at the 0.05 alpha level with an estimated drop out of $10 \%$. According to sample size calculation results, it was seen that 20 subjects per group were required. The level of the significance was accepted as $\mathrm{P}<0.05$ for all tests.

\section{RESULTS}

Altogether, 90 patients (44 females, 46 males) took part in the study and their respective data were analyzed. Characteristics of participants included in each group are presented in Table 1 and were homogenous for age, body weight, body height, smoking habits, and pain duration at baseline.

Tests of within-subject contrasts indicated that a general improvement was found in the intervention groups and such improvement was increasing over time reporting statistically significant differences among the groups, for all the aforementioned outcomes, excepted for the weight and VAS. Specifically, in these variables, statistically significant differences were found only between pain at rest during the day and pain at night $(\mathrm{P}=0.007)$ and between results, 95\% confidence interval and significance level are summarized in Table 2 . The manual therapy and exercise groups showed statistically significant greater improvements in pain intensity at night and at rest compared with the other groups at weeks 4 and $8(\mathrm{P}<0.05)$. There were no statistically significant differences in pain intensity among participants in the other groups $(\mathrm{P}>0.05)$.

No significant differences were found between participants in all groups in terms of stabilization, strength and functionality $(\mathrm{P}>0.05)$ (Tables 3, 4 and Figures 6, 7).

At baseline, medical history data of all patients were collected before enrollment. All the cases were stable at baseline. No adverse events or aggravation was observed in these patients. The physiotherapist who carried out the intervention was told to complete a list of attendance at the end of each session where adverse events were collected. There was no aggravation of symptoms in the lumbar spine.

\section{DISCUSSION}

LBP is a very common seen problem in clinics. Because there is no consensus regarding the best treatment for management of LBP, other alternative treatment options need to be explored for pain relief, increase of movement, and cure of the condition. Kinesio Taping is very effective in short term for the treatment of LBP; and manual therapy and exercise are also effective treatments as shown in literature. In this

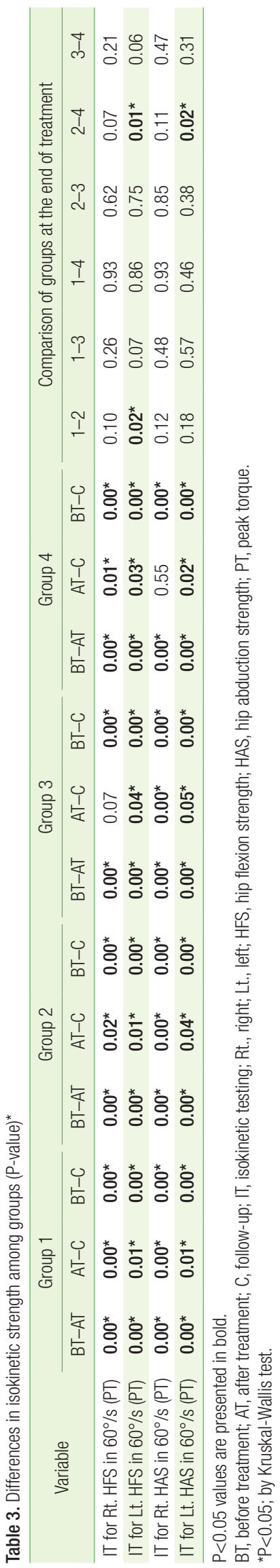


Table 4. Comparison of side plank test and Oswestry results among groups

\begin{tabular}{|c|c|c|c|c|c|c|c|}
\hline Groups & Category & $\begin{array}{l}\text { Sideplank_AT- } \\
\text { sideplank_BT }\end{array}$ & $\begin{array}{l}\text { Sideplank_C- } \\
\text { sideplank_AT }\end{array}$ & $\begin{array}{l}\text { Sideplank_C- } \\
\text { sideplank_BT }\end{array}$ & $\begin{array}{l}\text { Oswestry_AT- } \\
\text { Oswestry_BT }\end{array}$ & $\begin{array}{l}\text { Oswestry_C- } \\
\text { Oswestry_AT }\end{array}$ & $\begin{array}{l}\text { Oswestry_C- } \\
\text { Oswestry_BT }\end{array}$ \\
\hline \multirow[t]{2}{*}{ Group 1} & Z-value & $-4.020^{*}$ & $-4.046^{*}$ & $-4.286^{*}$ & $-4.157^{\star}$ & $-2.095^{\star}$ & $-4.206^{\star}$ \\
\hline & Asymp. Sig. (2-tailed) & 0.000 & 0.000 & 0.000 & 0.000 & 0.036 & 0.000 \\
\hline \multirow[t]{2}{*}{ Group 2} & Z-value & $-3.731^{*}$ & $-3.833^{*}$ & $-4.286^{*}$ & $-4.292^{*}$ & $-2.677^{\star}$ & $-4.288^{\star}$ \\
\hline & Asymp. Sig. (2-tailed) & 0.000 & 0.000 & 0.000 & 0.000 & 0.007 & 0.000 \\
\hline \multirow[t]{2}{*}{ Group 3} & Z-value & $-3.824^{*}$ & $-1.419^{*}$ & $-3.920^{*}$ & $-3.974^{*}$ & $-2.445^{\star}$ & $-4.017^{*}$ \\
\hline & Asymp. Sig. (2-tailed) & 0.000 & 0.156 & 0.000 & 0.000 & 0.014 & 0.000 \\
\hline \multirow[t]{2}{*}{ Group 4} & Z-value & $-3.922^{*}$ & $-3.622^{*}$ & $-3.621^{*}$ & $-3.623^{*}$ & $-3.036^{*}$ & $-3.624^{*}$ \\
\hline & Asymp. Sig. (2-tailed) & 0.000 & 0.000 & 0.000 & 0.000 & 0.002 & 0.000 \\
\hline
\end{tabular}

AT, after treatment; BT, before treatment; C, follow-up 4 weeks; Asymp. Sig., asymptotic significance. *Based on positive ranks; by Wilcoxon signed ranks test.

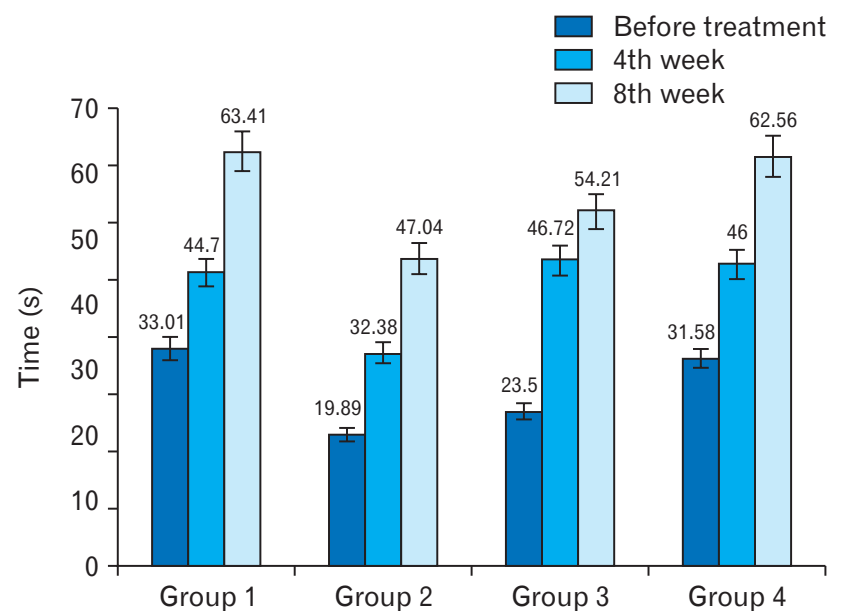

Figure 6. Side plank test result differences among groups.

study, the effects of four different treatment approaches consisting of Kinesio Taping, manual therapy and reflex therapy versus spinal exercises on the pain, disability, strength, stabilization, and functionality were investigated in the patients with chronic LBP. Based on the findings, all programs were found sufficiently effective in decreasing pain and improving strength and functional levels.

In our study, we found that all groups showed a decrease in pain at the end of treatment compared to the baseline level. Reduction in pain was the most important criterion in our research, because people refrain from moving as soon as they feel pain, which also leads them to feel bad about themselves. Decreased pain showed the effectiveness of treatment. The Kinesio Taping and reflex therapy groups showed a decrease in pain during activity and relaxation right after the treatment. This outcome resulted in an increase in their exercise tolerance. During treatment, the values for pain at night were not as high as those were for pain during activity and at rest, while these values before treatment were higher. At the second and third evaluations, we were able to see that all values for pain at night were reported as zero; and therefore, we can conclude that this was a parameter showing the fastest improvement. Pain at night causes the person to wake up several times during the night or change positions, which adversely affects the

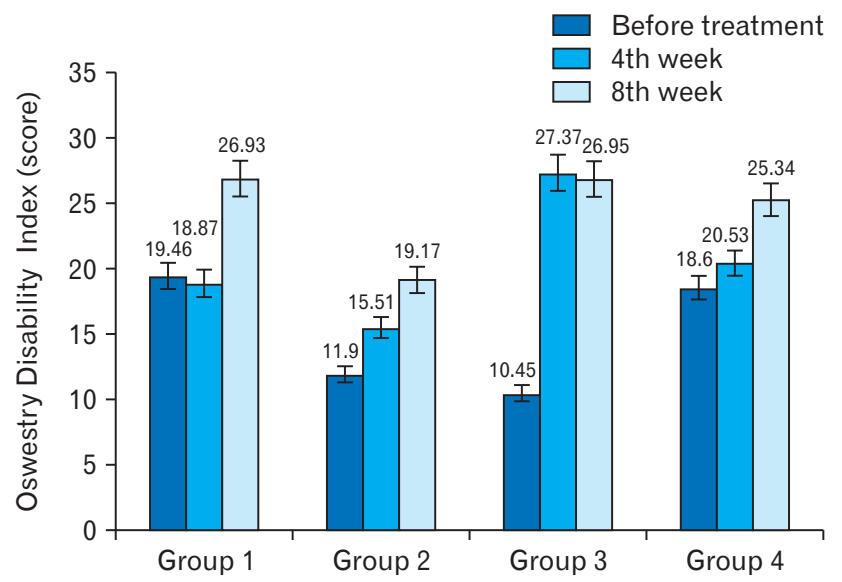

Figure 7. Oswestry Disability Index score differences among groups.

performance and psychological status during the day. All applications used in this research study, when combined with exercise, allowed patients to feel more functionally and psychologically active and led to shorter periods of returning to work compared to the other groups.

Goldby et al..$^{5)}$ stated that as a component of musculoskeletal physiotherapy a spinal stabilization program was more effective than manually applied therapy in treating chronic low back disorders over time. Both manual therapy and the spinal stabilization program are significantly effective in pain reduction. Koldas et al. ${ }^{4)}$ investigated the effects of three therapeutic approaches (aerobic exercise+home exercise, physical therapy+home exercise and home exercise only) to treat chronic PBP on pain levels, spinal mobility, disability, psychological state, and aerobic capacity. Their results showed that all of the three therapeutic approaches were effective in diminishing pain and thus increasing aerobic capacity in patients with chronic LBP. However, physical therapy+home exercise were found to be more effective in reducing disability and psychological disturbances. As we investigated the functional status, our results were found to be in agreement with the literature data, and exercises, including flexibility exercises, were found to be an essential part of the treatment program. Our Oswestry Disability Index results showed improvements at the second and third assessments, which indicated that patients' functionality quickly im- 
proved after treatment, allowing the patients to feel less pain and be more functional when performing activities of daily living.

Spinal mobilization and manipulation have different mechanisms of action on pain and movement limitation. ${ }^{6-9)}$ For instance, mobilization intends to increase soft tissue extensibility and joint mobility using low velocity passive force to achieve pain modulation via reduced hyperexcitability of muscle spindles and endogenous pain inhibition in the central nervous system. ${ }^{26)}$ Manipulation applies a sudden thrust that intends to make cracking sound to induce cavitation of a joint to achieve improvement of joint mobility and pain modulation via intradiscal pressure changes. ${ }^{29)}$ In addition, spinal mobilization generally requires longer treatment time, gentler touch by the clinicians and a more comfortable position for the patient than manipulation, which might lead to different effects between these manual therapy techniques. $^{30)}$

Marshall and Murphy ${ }^{16)}$ assessed lumbopelvic muscle activity during various core stability exercises with and without a Swiss ball. The researchers reported that although there was an evidence suggesting that the Swiss ball provided a training stimulus for the rectus abdominis, the relevance of this change to core stability training required further research because the focus of stabilization training was on minimizing rectus abdominis activity. A significant increase in rectus abdominis muscle strength was also found in our study in all groups at the end of treatment. The results showed that core stability exercises with and without a Swiss ball lessened pain and reduced disability. Segmental stabilization was superior to superficial strengthening for rectus abdominis muscle strength. Superficial strengthening did not improve transversus abdominis muscle activation capacity. In addition to these results, Jull and Richardson ${ }^{17)}$ reported that recent research on muscle dysfunction in patients with LBP led to discoveries of impairments in deep muscles of the trunk and back. These muscles have a functional role in enhancing spinal segmental support and control. The muscle impairments were not related to the strength but rather to the problems in motor control. These findings call for a different approach in therapeutic exercise, namely, a motor learning exercise protocol. ${ }^{17)}$ This specific exercise approach has an initial focus on retraining the co-contraction of the deep muscles (i.e., the transversus abdominis and lumbar multifidus). Initial clinical trials pointed to the effectiveness of this approach in patients with both acute and chronic LBP in terms of reducing the neuromuscular impairment and controlling pain. These studies showed that Swiss ball exercises were effective for LBP management and our results are in agreement with these findings.

Ferreira et al. ${ }^{8)}$ compared effects of general exercise, motor control exercise, and manipulative therapy on the function and perceived effect of intervention in patients with chronic back pain. The authors found that motor control exercise and spinal manipulative therapy produced slightly better short-term, but not medium- or long-term, effects in patients with chronic non-specific back pain. Senna and Machaly ${ }^{30)}$ determined the effectiveness of maintenance spinal mobilization therapy in long-term reduction of pain and disability levels as- sociated with chronic low back conditions after an initial phase of treatments. They stated that spinal mobilization therapy was effective for the treatment of chronic non-specific LBP. To obtain a long-term benefit, they suggested using maintenance spinal mobilization after the initial intensive manipulative therapy. Similar to this, we observed that the manipulative therapy group showed faster pain relief. In our study, some patients who did not exercise regularly had an increased level of pain during 1 month after the treatment.

There were reductions in pain during activity, at rest and at night after treatments and at 4 weeks after the follow-up in all treatments in the present study. In earlier studies the reduction was greater- $49.0 \%$ in the spinal mobilization therapy group after a 7-day residential manual therapy program. ${ }^{3)}$ Differences in the treatment programs for LBP could explain why the reduction observed was lower in this study. While in some studies, ${ }^{4,8,9,30)}$ the efficacy of exercise treatment was demonstrated in comparison with the spinal exercise group, in this study, there was no placebo group because it was considered unethical to use a placebo group in such design.

A limited number of trials have assessed non-pharmacological treatments for the management of LBP and to our knowledge, this is the first randomized trial assessing the effectiveness of different kind of physiotherapy approaches in addition to a supervised spinal stabilization exercise program in this population. Our primary analysis showed that adding supervised spinal stabilization exercise program did not result in significant or meaningful advantages in pain severity over the course of 4 weeks. Secondary analyses showed that at the end of the 4-week treatment phase and a 4-week follow-up, group differences favored the addition of either Kinesio Taping, reflexology, or spinal manipulative therapy by $8 \%, 4 \%$, and $4 \%$ points, respectively. Group differences following the end of treatment were smaller ( $0 \%$ to $3 \%$ points) and favored Kinesio Taping plus stabilization exercise program alone over the combined treatment groups. Differences in secondary patient-rated outcomes were small and did not show any clear pattern in favor of one treatment over another. An exception was the satisfaction with care, where participants in all soft tissue mobilization+stabilization exercise and Kinesio Tape+stabilization exercise and reflexology+stabilization exercise consistently reported greater satisfaction than those in the stabilization exercise group throughout the 8-week course.

Our trial has several strengths, including four different types of management, including homogenous groups and a rigorous design with emphasis on internal validity. We also had excellent engagement and follow-up rates. The findings of this study should be interpreted in the context of its limitations. Limitations of the study include inability to blind patients and providers to the nature of the interventions. First, the duration of intervention was brief (i.e., 4 weeks), thus the 'dose' of the treatment might have been insufficient to create quantitatively observable changes in some of the outcomes measured. Second, the sample size was small. The low number of patients included in this study is a limitation that could have decreased the power of statistical analysis. However, various methods of analysis were conducted to complement and strengthen the quantitative findings obtained from 
the small sample size of the study. The results from each analysis validated each other in several points and provided stronger evidence for the conclusions. In addition, utilization of both subjective and objective data collection and analysis techniques to compare/complement/ validate results against each other should be used to increase the validity of the research findings. Therefore, more randomized controlled trials will be needed to establish efficacy and, subsequently, treatment effectiveness.

Adding supervised stabilization exercise program alone does not appear to improve pain or disability outcomes in either short- or longterm in patients with chronic LBP, but did enhance satisfaction with care. On the other hand, Kinesio Taping decreased immediate pain levels. When considered together, the findings of this study suggest that a 4-week program with different approaches is a viable treatment option for patients with chronic LBP. Therefore, we believe that stabilization exercises should be included in exercise prescription by physical therapists. The cost-effectiveness of these interventions needs to be assessed.

\section{CONFLICT OF INTEREST}

No potential conflict of interest relevant to this article was reported.

\section{ORCID}

Gamze Senbursa: https://orcid.org/0000-0002-7968-2176

Nihan Ozunlu Pekyavas: https://orcid.org/0000-0003-0603-5688

Gul Baltaci: https://orcid.org/0000-0002-6513-2499

\section{REFERENCES}

1. Atchison JW, Taub NS, Cotter AC, Tellis A. Complementary and alternative medicine treatments for low back pain. Phys Med Rehabil 1999;13:561-86.

2. Katz JN. Lumbar disc disorders and low-back pain: socioeconomic factors and consequences. J Bone Joint Surg Am 2006;88 Suppl 2:21-4.

3. Hazard RG. Low-back and neck pain diagnosis and treatment. Am J Phys Med Rehabil 2007;86(1 Suppl):S59-68.

4. Koldas Dogan S, Sonel Tur B, Kurtais Y, Atay MB. Comparison of three different approaches in the treatment of chronic low back pain. Clin Rheumatol 2008;27:873-81.

5. Goldby LJ, Moore AP, Doust J, Trew ME. A randomized controlled trial investigating the efficiency of musculoskeletal physiotherapy on chronic low back disorder. Spine (Phila Pa 1976) 2006;31:1083-93.

6. Chou R, Qaseem A, Snow V, Casey D, Cross JT Jr, Shekelle P, et al. Diagnosis and treatment of low back pain: a joint clinical practice guideline from the American College of Physicians and the American Pain Society. Ann Intern Med 2007;147:478-91.

7. Hu X, Chen N, Yang G, Chai Q, Trevelyn E, Lorenc A, et al. Integrated treatment for low back pain: a systematic review. Eur J Integr Med 2013;5:572.

8. Ferreira ML, Ferreira PH, Latimer J, Herbert RD, Hodges PW, Jennings $\mathrm{MD}$, et al. Comparison of general exercise, motor control exercise and spinal manipulative therapy for chronic low back pain: a randomized trial. Pain 2007;131:31-7.

9. Ulger O, Demirel A, Oz M, Tamer S. The effect of manual therapy and exercise in patients with chronic low back pain: double blind randomized controlled trial. J Back Musculoskelet Rehabil 2017;30:1303-9.

10. Dalal K, Elanchezhiyan D, Das R, Dalal D, Pandey RM, Chatterjee S, et al. Noninvasive characterisation of foot reflexology areas by swept source-optical coherence tomography in patients with low back pain. Evid Based Complement Alternat Med 2013;2013:983769.

11. McCullough JE, Liddle SD, Sinclair M, Close C, Hughes CM. The physiological and biochemical outcomes associated with a reflexology treatment: a systematic review. Evid Based Complement Alternat Med 2014;2014:502123.

12. Ramirez-Velez R, Hormazabal-Aguayo I, Izquierdo M, Gonzalez-Ruiz K, Correa-Bautista JE, Garcia-Hermoso A. Effects of Kinesio Taping alone versus sham taping in individuals with musculoskeletal conditions after intervention for at least one week: a systematic review and meta-analysis. Physiotherapy 2019;105:412-20.

13. Castro-Sanchez AM, Lara-Palomo IC, Mataran-Penarrocha GA, Fernandez-Sanchez M, Sanchez-Labraca N, Arroyo-Morales M. Kinesio Taping reduces disability and pain slightly in chronic non-specific low back pain: a randomised trial. J Physiother 2012;58:89-95.

14. Kim CH, Kim AR, Kim MI, Kim SH, Yoo HJ, Lee SH. The efficacy of Kinesio Taping in patients with a low back pain. J Korean Acad Fam Med 2002;23:197-204.

15. Hodges PW. Core stability exercise in chronic low back pain. Orthop Clin North Am 2003;34:245-54.

16. Marshall PW, Murphy BA. Core stability exercises on and off a Swiss ball. Arch Phys Med Rehabil 2005;86:242-9.

17. Jull GA, Richardson CA. Motor control problems in patients with spinal pain: a new direction for therapeutic exercise. J Manipulative Physiol Ther 2000;23:115-7.

18. Quinn F, Hughes CM, Baxter GD. Reflexology in the management of low back pain: a pilot randomised controlled trial. Complement Ther Med 2008;16:3-8.

19. Mouzarou A, Psatha A, Apostolopoulos P, Siori I, Karamichali E, Kyriakides M. Case reports of three patients suffering from low back pain treated with reflexology. Eur J Integr Med 2010;2:238.

20. Paoloni M, Bernetti A, Fratocchi G, Mangone M, Parrinello L, Del Pilar Cooper M, et al. Kinesio Taping applied to lumbar muscles influences clinical and electromyographic characteristics in chronic low back pain patients. Eur J Phys Rehabil Med 2011;47:237-44.

21. Schulz KF, Altman DG, Moher D; CONSORT Group. CONSORT 2010 statement: updated guidelines for reporting parallel group randomised trials. J Clin Epidemiol 2010;63:834-40.

22. Jensen MP, Karoly P. Self-report scales and procedures for assessing pain in adults. In: Turk DC, Melzack R, editors. Handbook of pain assessment. 2nd ed. New York (NY): Guilford Press; 2001. p. 15-34.

23. Claiborne TL, Timmons MK, Pincivero DM. Test-retest reliability of cardinal plane isokinetic hip torque and EMG. J Electromyogr Kinesiol 2009;19:e345-52.

24. Imai A, Kaneoka K. The relationship between trunk endurance plank tests and athletic performance tests in adolescent soccer players. Int J Sports Phys Ther 2016;11:718-24.

25. Yakut E, Duger T, Oksuz C, Yorukan S, Ureten K, Turan D, et al. Valida- 
tion of the Turkish version of the Oswestry Disability Index for patients with low back pain. Spine (Phila Pa 1976) 2004;29:581-5.

26. Gomes-Neto M, Lopes JM, Conceicao CS, Araujo A, Brasileiro A, Sousa $\mathrm{C}$, et al. Stabilization exercise compared to general exercises or manual therapy for the management of low back pain: a systematic review and meta-analysis. Phys Ther Sport 2017;23:136-42.

27. Berry G, Svarovska B. Report on a membership audit of the Association of Chartered Physiotherapists in Reflex Therapy (ACPIRT). Complement Ther Clin Pract 2014;20:172-7.

28. Kase K, Wallis J, Kase T. Clinical therapeutic application of the Kinesio
Taping method. 2nd ed. Tokyo: Ken Ikai Co.; 2003

29. Balthazard P, de Goumoens P, Rivier G, Demeulenaere P, Ballabeni P, Deriaz O. Manual therapy followed by specific active exercises versus a placebo followed by specific active exercises on the improvement of functional disability in patients with chronic non specific low back pain: a randomized controlled trial. BMC Musculoskelet Disord 2012;13:162.

30. Senna MK, Machaly SA. Does maintained spinal manipulation therapy for chronic nonspecific low back pain result in better long-term outcome? Spine (Phila Pa 1976) 2011;36:1427-37. 